\title{
The Electronic Laboratory Journal: A Collaborative and Cooperative Learning Environment for Web-Based Experimentation
}

\author{
GEORGIOS JOHN FAKAS ${ }^{1}$, ANH VU NGUYEN ${ }^{2} \&$ DENIS GILLET ${ }^{2}$ \\ ${ }^{1}$ Department of Computing and Mathematics, Manchester Metropolitan University, Chester \\ Street, Manchester, M1 5GD, UK (E-mail: g.fakas@nmu.ac.uk); ${ }^{2}$ École Polytechnique \\ Fédérale de Lausanne (EPFL) ME-EPFL - Ecublens, CH 1015, Lausanne, Switzerland \\ (E-mail:denis.gillet@epfl.ch)
}

\begin{abstract}
Numerous tools have been developed for supporting the collaboration between students in education, tools that mainly include facilities for sharing documents and enabling discussions. However, these environments do not emphasize the use of facilities that sustain collaborative work in the framework of remote experimentation carried out by a group of students located at different places. The Electronic Laboratory Journal (eJournal) paradigm proposed in this paper is a collaborative and cooperative environment for Web-based experimentation in engineering education. The eJournal enhances the traditional laboratory journal, by providing a group of students with Web-based tools to collect, annotate, organize and share the data chunks necessary to complete their experimentation assignments. The data chunks, called fragments, may be composed of numerous objects of any format, such as text, images, graphics, manuscripts, measurement logs or experimental results. Fragments can be uploaded from local disks or imported from Web components. The eJournal also handles the submission of results to the educators and facilitates remote supervision, assistance and tutoring of the students. The eJournal paradigm is currently assessed at the School of Engineering, the École Polytechnique Fédérale de Lausanne (EPFL), in the framework of hands-on experimentation activities focusing on remote manipulation of real setups and Webbased simulation. This paper presents the eJournal environment, its application and its evaluation as an enabling Web-based application for flexible learning.
\end{abstract}

Key words: cooperative learning, collaborative learning, distance learning, knowledge engineering, remote experimentation, World Wide Web

\section{Introduction}

The rapid development of computer networking and the Internet in the last decade has provided new possibilities and also new challenges for designing and deploying distributed and collaborative applications. One of the areas that has benefited from such technologies is education, where significant efforts have been directed towards the design and implementation of asynchronous learning network environments for distance and flexible Web-based learning (Latchman 
et al., 1998). Especially in engineering curricula, a useful and interesting trend to support learning is to expand the available educational resources by providing virtual and real experimentation facilities (Gillet and Fakas, 2001). Web-based experimentation turns to be a key feature in the deployment of e-Learning solutions for engineering education. It offers a tremendous opportunity to add flexibility in traditional curriculum by providing students with versatile access to the learning material from both a time and a location perspective.

Important ingredients in engineering education are practical laboratory activities carried out usually by students working in pairs. Hands-on activities are recognized as an efficient approach for students to solve real world problems (Roschelle et al., 1999; Schmid, 1999; Gillet et al., 2000; Armstrong and Perez, 2001). This introduces two requirements for building Web-based environments that support virtual or real experimentation. First, the Web environment must provide the students with interactive content for performing hands-on activities allowing multiple trial-and-error cycles. Second, the Web environment must support collaborative activities. Collaboration is an essential ingredient in the recipe to create an effective learning environment in engineering education, as it provides students with the opportunity to discuss, argue, and exchange information or knowledge. The collaboration between students working actively in small groups can help them to work more productively in the laboratory and also learn more easily. The literature seems to be supporting that the social interaction amongst learners plays an important part in the learning process. In fact, it can have a significant impact on learning outcomes (Harasim, 1989; Berge and Collins, 1995; Eastmond and Ziegahn, 1995; Jonassen et al., 1995; Agostinho et al., 2001). The first requirement is usually fulfilled through access to interactive course content that can take the form of (i) simulations driven by Java applets, CGI scripts, and/or (ii) components that connect to a remote server, which controls real physical devices (Gillet et al., 2000; Tan et al., 2000; Gillet and Fakas, 2001). The Web-based engineering learning environments should support the integration, and thus the interaction, of heterogeneous components since it allows the sharing of laboratory resources from different institutions. The second requirement is usually fulfilled through the provision of communication tools, such as chat and threaded discussion, and a shared workspace such as BSCW for sharing documents (Appelt and Mambrey, 1999; Klöckner, 2000). In summary, Web-based engineering learning environments should provide tools to fulfill these two essential requirements.

\subsection{ELECTRONIC JOURNALS AS COLLABORATIVE TOOLS IN ENGINEERING EDUCATION}

Electronic journals for personal use have a lot in common with paper journals. In some cases, the journals can be called notebooks. Like 
paper journals, the electronic journals serve as work diaries. However, the electronic content is easier to archive, to search, to browse and to copy; thus electronic journals encourage further exploitation of their content.

Laboratory journals take a privileged place in science and engineering practices (McCormack et al., 1991; Myers et al., 1996). The laboratory notebook serves as a chronological repository for experimentation planning and realization. It enables to keep operational details, collected data, thoughts and further objectives to sustain the discovery or the validation activities. The benefit of taking electronic notes in science and engineering is even greater when the journal is shared among a group of co-workers. Hence, it seems also appropriate to use it in engineering education where such scientific methodology has also to be acquired.

\subsection{NOVEL CONTRIBUTIONS}

In this paper, we define, validate and evaluate approaches and resources that enable collaborative work in the framework of Web-based experimentation carried out together by students in a flexible context. We reproduce in a flexible way the teamwork scheme traditionally applied on campus by students running laboratory experiments or interactive simulation sessions together. The Electronic Laboratory Journal metaphor is introduced as a dedicated component of a Web-based learning environment to support over the Internet collaborative and cooperative activities within groups of students committed to complete together a specific experimentation assignment. The Laboratory Journal metaphor was chosen, as we believe that engineering students are familiar with this concept and it will be easy for them to use and conceptualize. The eJournal is an electronic and extended version of the traditional journal where students can maintain and share data in order to collaborate as well as document their practical work. It includes many advanced functionalities for sustaining the learning and collaboration process in flexible hands-on activities. The eJournal also enables educators to supervise students' progresses and provide asynchronous support. Finally, the eJournal provides metrics for the evaluation of the learning modalities.

The eJournal is integrated and validated as a component of the eMersion environment. The eMersion environment is a Web-based experimentation environment developed at EPFL. The eMersion environment is based on a cockpit metaphor and supports hands-on experimentation through remote manipulation of physical laboratory devices and/or computer simulation tools. The environment provides the students with the possibility to carry out experimentation in a flexible way, i.e. the students can follow different learning modalities (Sire et al., 2003) to perform multi-session experiments. Within the eMersion environment, the eJournal not only serves as a shared 
artifact that facilitates the interaction and collaboration process among students, but also as an exchange platform between integrated Web-based components.

\section{Related work}

Numerous successful Web-based collaborative environments exist, which are used in education and in research in general that support remote experimentation. However, there are not any open and also dedicated collaborative tools that support the whole life-cycle of the execution of group-based experimentation coursework, i.e. to include remote experimentation, collaboration, cooperation, documentation, coursework deliverables submission and educator supervision and assistance facilities.

\subsection{RELATED WORK IN ELECTRONIC JOURNALS}

The Electronic Notebook (EN) which is a similar tool, developed at Pacific Northwest National Laboratory, Lawrence Berkeley National Laboratory and Oak Ridge National Laboratory in the US Department of Energy (Geist and Nachtigal, 2001; Myers et al., 2003), is a Web-based version of a paper research notebook that facilitates the electronic recording of information (such as sketches, text, equations, images, graphs, signatures, and other data) and also allows geographically distributed partners to collaborate by sharing and recording ideas, data and events of joint experiments and research programs. Another widely used cooperative tool in the academic community is the Basic Support for Cooperative Work (BSCW) system (Appelt and Mambrey, 1999; Klöckner, 2000), which facilitates the collaboration of partners in order to perform their activities. BSCW is for general use and does not provide any dedicated facilities for remote laboratory experimentation. Another interesting learning environment that emphasizes projectbased science learning is the Learning Through Collaborative Visualization (CoVis), developed at Northwestern University (Gomez and Gordin, 1996), which is used in 40 high school science classrooms, and supports communication and visualization tools that enable communication and collaboration among learners.

This overview shows that laboratory journals in education are mostly restricted to the collection of documents into shared workspaces, or to the collaborative editing of text-oriented pages that may refer to external documents collected by other means, such as Web links if they reside on a Web server. Those environments are usually for general use and do not provide dedicated facilities for hands-on experimentation. This raises questions about the way laboratory journals should be integrated into engineering learning 
scenarios, in which the hands-on activities and collaborative activities play an important role.

\subsection{OTHER RELATED WORK}

The I-Labs project is a cooperation between the Stanford Center for Innovations in Learning (http://scil.stanford.edu), USA and the LearningLab Lower Saxony (http://www.learninglab.de/i_labs/), Hanover, Germany. The Stanford team focuses on the optical experiments in physics education, and the LearningLab team develops mechatronics experiments for engineering education. By working with an online lab students shall learn to program, maintain and supervise remote devices. In Hanover, a remote experiment for picture generation by laser deflection has been developed (Böhne et al., 2004). The system facilitates students to communicate synchronously with each other via audio, video, text chat, and desktop sharing utilities provided by external applications such as Macromedia Flash MX, NetMeeting, and VNC. Asynchronous communication is supported using BSCW (http:// bscw.gmd.de). However, although this approach provides students with the possibility to perform the hands-on activities remotely, the students cannot follow multi-session experiments; for instance, they cannot complete parts of the experiment at school, and then pursue the remainder of the work at home using the same environment.

At Stanford University, the Centre for Design Research has studied for many years the implementation of engineering product development teams at work in academic and corporate settings and they introduced the concept of Problem Based Learning (PBL), which is based on team-based, distance learning techniques (Leifer, 1997). In this context, the ME310 course, a real world learning experience, supports design teams, professional coach, corporate liaisons and faculty advisor (ME310).

Collaboratories are also related environments that could be based on the use of tools like eJournals or ENs. They use collaborative technologies (e.g. interaction between colleagues, access to remote instruments, and sharing of data and computer resources) in order to support distributed scientific research. Several successful Collaboratories have been developed, e.g. the Upper Atmospheric Research Collaboratory (Olson et al., 1998), the Collaboratory to support Distributed Science (the HIV/AIDS Research) (Olson et al., 2002), the Research Collaboratory for Structural Bioinformatics, which manages the Protein Data Bank (Berman et al., 2000) and the Environmental Molecular Sciences Laboratory (Kouzes et al., 1996). Future directions of Collaboratories are the emerging Cyberinfrastructures, proposed by (Atkins et al., 2003) and (Arzberger and Finholt, 2002) that will radically empower scientific and engineering research and allied education by integrating together data, instruments, scientists, networks, software, and high-performance computing. Trends in 
scientific research based on distributed project teams and large-scale coordination of efforts thought Collaboratories and Grid-based virtual organizations (Foster, 2002) emphasize the use of eJournals and ENs systems.

\section{A web-based collaborative learning environment for engineering education}

\subsection{THE LEARNING SETTINGS}

In engineering education, the practical activities are as important as the theoretical ones. In the spirit of flexible learning (Gillet, 2003), students have the possibility of carrying out an experiment at any time and from any location, thus benefiting from a more effective cognitive experience. In other words, the student is provided with the possibility to follow different learning modalities. The modalities vary according to the presence of teaching assistants (TA), and according to the student's location. When group members work together in presence of TA, they are in a face-to-face (f2f) condition. The location can be a laboratory with full access to physical devices (local condition), a computer room on the campus (remote-campus condition), a student's home or any other place (remote-home condition). In the last two conditions students remotely access the physical laboratory devices and/or computer simulation tools. Students can perform multi-session experiments; that means, for instance, they can do the first part of an experiment at school, and pursue the rest of it at home. Figure 1 shows students working in the laboratory (f2f modality) and interacting directly with the TA.

The academic year at EPFL is divided into a winter and a summer term. We have iteratively designed and deployed our Web-based learning environment since the 2000 winter term. The environment has been used for hands-on experimentation in Automatic Control, Biomechanics and Fluid

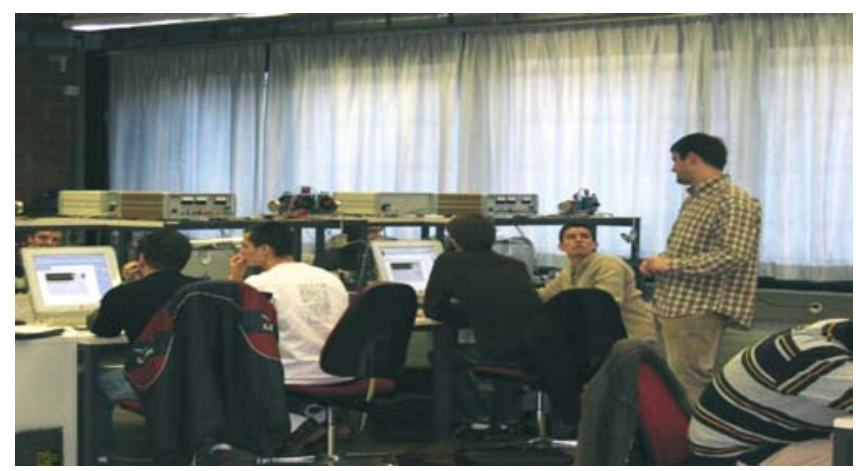

Figure 1. F2f learning modality. 
Mechanics. However, only the hands-on activities in Automatic Control have been evaluated.

\subsection{THE COCKPIT ENVIRONMENT}

A comparative study has been carried out in Automatic Control, Biomechanics and Fluid Mechanics to determine the most common features required for completing typical experimentation assignments by students enrolled in the third and fourth year of the engineering curricula at EPFL. Students have also been observed in real laboratory conditions, by pedagogues, to understand their needs and interaction modes. In addition to dramatically improving effectiveness and reducing the development time, this concerted approach has led to a generic solution that can easily be deployed for educational purposes in other engineering domains. The Cockpit environment resulting from this comparative study as well as from the student observations contains all the components necessary to successfully complete laboratory assignments (Gillet et al., 2003). Those components are heterogeneous in the sense that they were developed using different technologies and may be located on different servers.

The main components are as follows

1. Experimentation component: it was developed as a Java applet and can be regarded as the interaction part that enables the actual realization of experiments. The interactions that can be sustained are mainly in the form of changes that the students can make to parameters or algorithms that affects the behaviors of the virtual model or of the real piece of equipment. The responses to the changes are displayed graphically in real-time.

2. SysQuake Remote component: it is a PHP application, which provides students with tools to carry out interactive design and analysis activities related to the experiment. It embeds advanced computational and graphical functionalities such as parameterized graphics, graphical representations, etc. The SysQuake Remote's script is Matlab compatible.

3. eJournal: it will be presented in the next sections.

4. Supporting components: include a clear statement of the module's objectives, relevant theory, such as short reminders or links to theoretical references, an experimental protocol, which corresponds to the step-by-step procedures required to perform the module, a description of the environment, including the experimental facilities (real or virtual) and the detail cockpit features, and any bibliography.

Figure 2 shows the Cockpit environment, which includes the components to carry out the automatic control modules, in the case of the tele-operation of an electrical drive. This drive is visualized in real-time within the cockpit using a Webcam. Details of the environment are presented in (Gillet et al., 2003). 


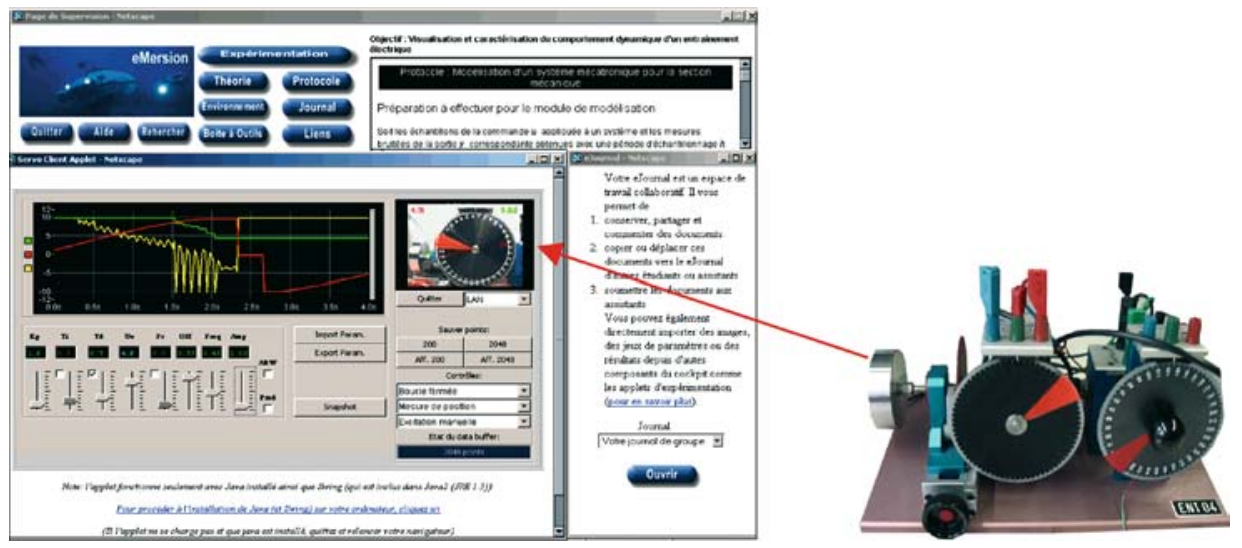

Figure 2. The cockpit environment.

\section{The eJournal}

The eJournal is an electronic and extended version of the traditional laboratory journal used by students during their laboratory sessions. Students can collaborate and cooperate by maintaining and sharing fragments, which are documents stored in the eJournal, in order to remotely document, complete and finally submit their practical teamwork. A number of requirements have been defined for the design of the eJournal. The first and most important requirement was to keep the system simple and easy to use by avoiding unnecessary functionalities. The eJournal is simply a dedicated environment to be used by a group of students to work on a specific team project and it does not include specialized collaborative functions, e.g. advanced discussion facilities, teleconferencing, calendaring, etc. A secondary requirement was to encapsulate and emphasize in the eJournal design, the whole cycle of experimentation teamwork; i.e. study of any required preparatory material, educators' supervision and tutoring, execution of work, and finally submission of results. A third requirement was that the eJournal should also support the data exchange among different components within the same Web-based experimentation environment. It serves as a persistent store for the experimental data input/output that are generated and/or used by different components in different steps of the experimentation cycle. Another important requirement was to develop an open and reusable system that can be integrated into other experimentation environments in engineering or even applied in any area in education where students have to perform group work.

A typical laboratory team project, which involves various resources that can or have to be accessed synchronously or asynchronously, is a succession of tasks carried out by a team of students. Common experimentation activities include study of preparatory information and readings of theoretical 
material, allocation of tasks between team members, definition of schedules, sharing of thoughts and contributions, discussions, educators' supervision, assistance and tutoring, use of interactive experimentation or simulation facilities, post processing of results and analysis, and finally, submission of results. The eJournal enables the Web-based execution of such activities by providing a common workspace for the laboratory assignments of each team.

The eJournal can be launched either as a component of the Cockpit environment or as a stand-alone Web application. The eJournal's main space looks like the mailbox of an email client, except that it does not contain email but rich-type data fragments (Figure 3). There are two different kinds of eJournal, one is for students and another is for the Educator (professor and TA). The latter is called the Educator's eJournal. The interface of Educator's eJournal is slightly different.

When a user logs on, the system detects the role of this user based on the entered username and password and then launches the appropriate eJournal.

The implementation of the eJournal is mainly based on the use of Java, JavaBeans, JSP and XML technologies. We also use MySQL relational database management to store the meta-information. So, we can achieve an interoperable and reusable system. The eJournal data can easily be interchanged with different applications, such as Web-based experimentation tools. These properties make the eJournal an open, reusable, scalable and multi-platform system.

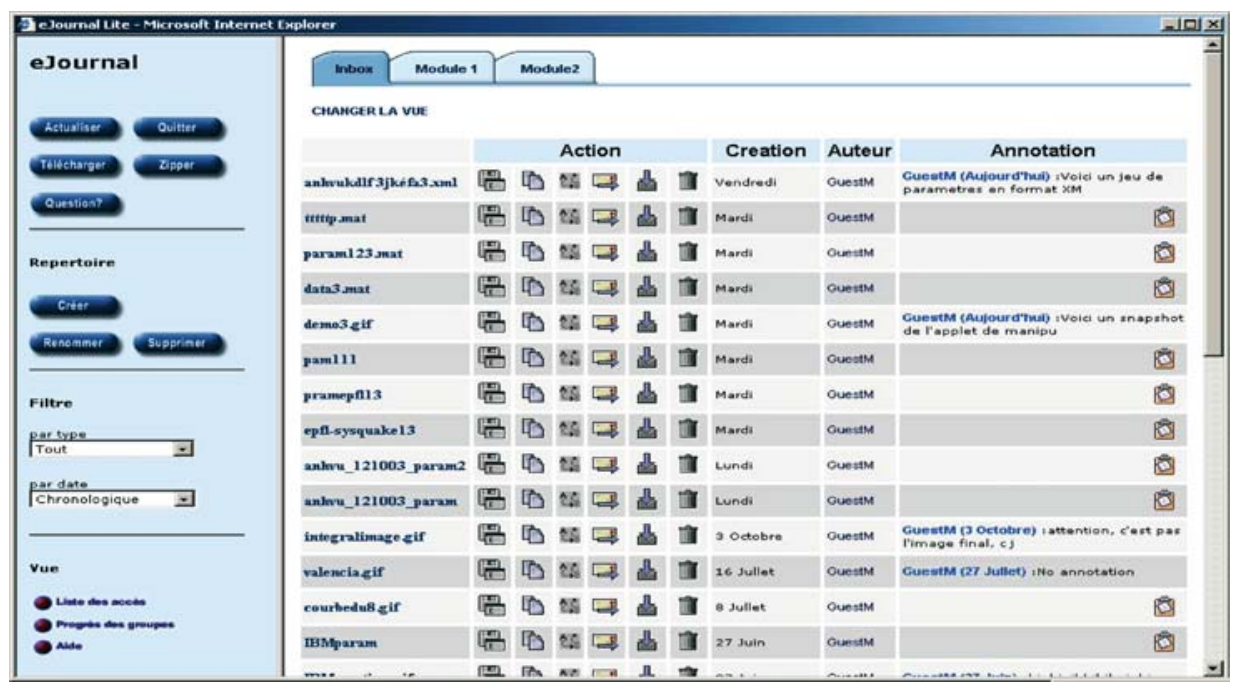

Figure 3. The eJournal user interface. 
The main functionalities of the eJournal can be grouped into different categories: Fragments collection, fragments exchange and awareness as described below.

\subsection{FRAGMENTS COLLECTION}

The fragments collected by students when carrying out the experimentation, following a protocol described in the cockpit, are stored in the student eJournal. Any fragment is typed, representing different kinds of data. Fragments with different types are handled differently. Many meta-data are used to describe a fragment. Those include fragment identification, fragment name, author, creation date, type, MIME type, size, module, task, etc. The fragments can be grouped into different folders, which allow students to better organize their data. For example, a student can create a folder for each module, and place all fragments used during the module realization in this folder. The fragments stored in the eJournal can be filtered based on different categories, such as date of creation, fragment type, module, or task performed. The fragments can be deleted temporarily (put to Trash) or definitely (physically deleted).

The eJournal is fully connected with the other software components for an automatic importing and exporting of various types of data. One can use the eJournal to upload fragments from local disks; can import/export snapshots, parameter sets and/or numerical results from/to other Cockpit components such as the Experimentation applet or SysQuake Remote (the data analysis component of the environment). This feature is important in the sense that the eJournal, as a laboratory journal, should support the experimental data input/output. This possibility plays a key role for sustaining the continuity of interaction in flexible hands-on and collaborative activities (Nguyen et al., 2004a).

The Educator's eJournal can only upload fragments from the local disk and receive fragments submitted from other students' eJournals.

Figure 4 shows the Educator's eJournal interface. In the left panel, there is a list of enrolled groups. In the right panel, one can see the submitted fragments (called pre-labs in the case of the automatic control modules) of the selected group and a list of cockpits, i.e. lab modules this group has to carry out. If the submitted fragments related to a given module are accepted, the TA can check it to allow further activities.

\subsection{FRAGMENTS EXCHANGE}

The eJournal provides a shared workspace, where students can store, retrieve, share and exchange the group fragments when performing the experiments. There are different workspaces. The private eJournal space can 


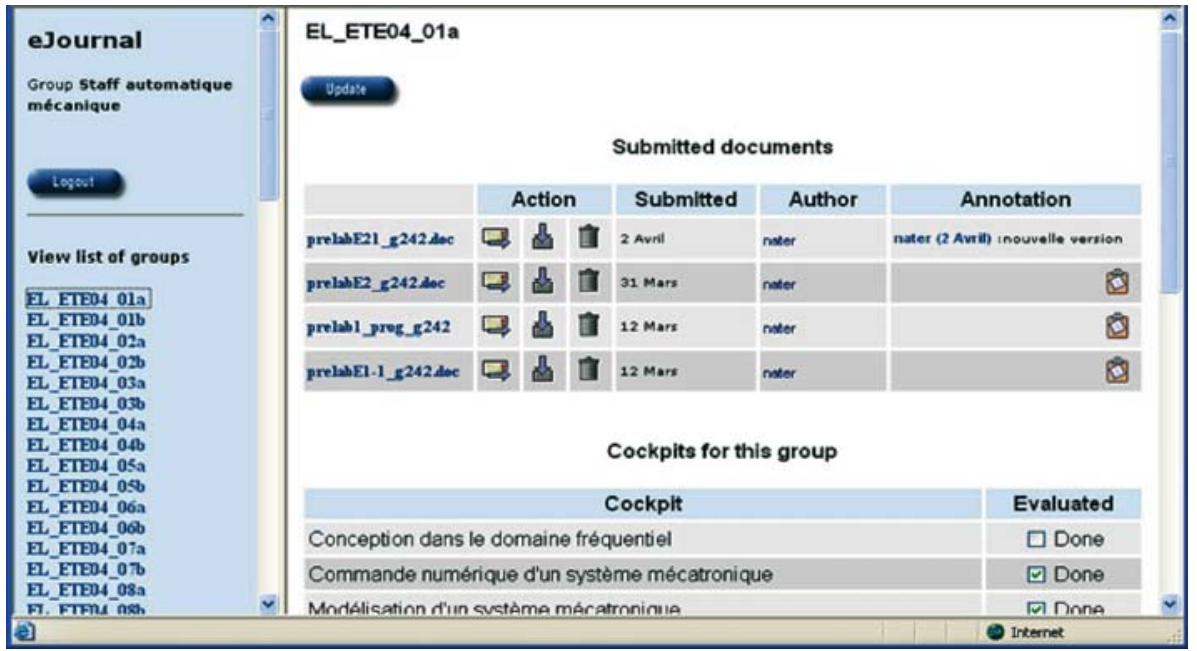

Figure 4. The educator eJournal interface.

only be read or modified by the owner (typically a group of two students). Other eJournal spaces can be accessed provided that the proper rights have been granted by their respective owners.

The Access Control List (ACL) mechanism defined by the eJournal provides the possibility for students to give permissions to members of other groups. The permissions are associated with the fragments. Two main permissions have been defined. Those are Visible (to allow users to view a fragment) and Copy-able (to allow users to copy a fragment from one folder to another folder in the same journal, or from one journal to another journal). We have defined some different kinds of users

1. Group members: have all permission over the group fragments

2. Class: Member of other groups (i.e. they have enrolled in the course to use the Cockpit environment but are not working in the same group)

3. Educators: Professors and TAs

4. Others: Other kinds of users such as Guest

The fragments can be annotated. Students can directly send fragments with associated annotations, or send questions with attached fragments to other groups or to the TA via an integrated email system. This mechanism can also be used for submission, and for contextualized help. For instance, students can send a question to a TA with an attached fragment. The fragment attached in such a case can make the question more understandable since it embeds the student's current experimental context.

In addition to supporting the exchange of fragments between students, the eJournal also supports, as mentioned previously, the exchange of fragments 
between Web components. The eJournal is a convenient medium to sustain the continuity of interaction and to support the collaboration among members of the learning community. This concept, which means that interruptions of activities across modalities are avoided as much as possible, is quite important in the context of flexible hands-on experimentation activities. Figure 5 represents an example of continuous interaction. The Experimentation component (a Java applet) exports experimental results to the eJournal, which will then be processed using the SysQuake Remote analysis component. Users do that simply by 'clicking buttons'. This means that, for instance, on campus, a student can click on the 'Export Results' button in the Experimentation console to save the experimental result as a fragment typed 'Results' in the group's eJournal. Later at home, the same student or another member of the group can click on this fragment in the eJournal to load automatically the SysQuake Remote console. In fact, depending on the type of fragment, an appropriate component is launched. The continuous interaction mechanism augments a lot the interaction process. Data are passed smoothly and naturally from one component to another. The requirement to use external applications for data sharing and exchanging is minimized. Users work with minimum discontinuity in all dimensions of interaction. As a consequence, the quality of the hands-on and collaborative works is much more improved (Nguyen et al., 2004a).

\subsection{AWARENESS}

Knowing the activities of other co-workers is a basic requirement for group interaction, which is the visible aspect of collaborations (Martínez et al., 2002). In a face-to-face condition, users find it naturally easy to maintain a sense of awareness about the activities of others. However, in other conditions, supporting spontaneous interaction is evidently much more difficult. To support effective collaboration, systems should provide group awareness, which is defined as "an understanding of the activities and progresses of
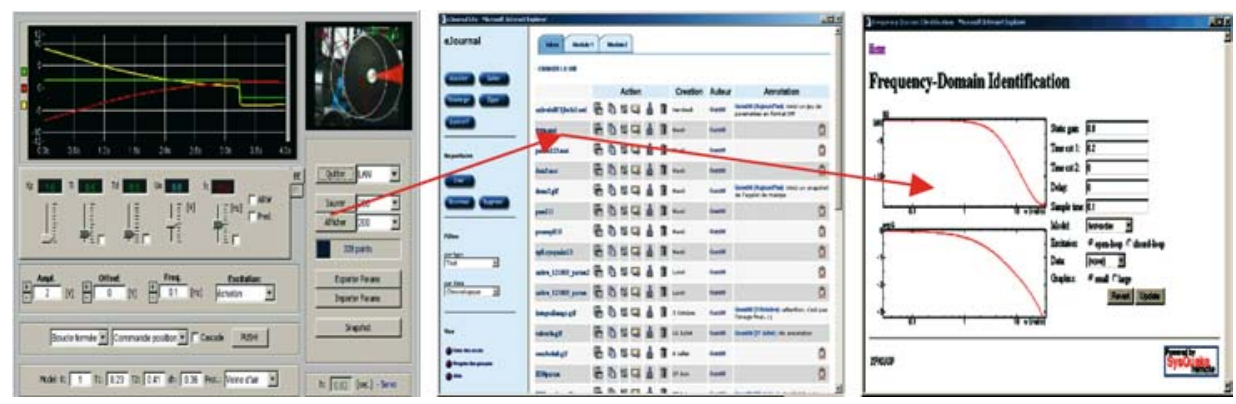

Figrue 5. Continuous interaction. 
others, which provides a context for your own activities" (Dourish and Bellotti, 1992). Various awareness mechanisms have been produced to support group awareness (Gutwin et al., 1996), such as tele-pointers, radarviews, or distortion-oriented lenses. Generally, the system gathers data about the students' interaction, and shows some visualization of this information to the user. It is then up to users to interpret the awareness and decide which actions to take.

The awareness plays an important role to facilitate the teaching and learning processes, especially in a flexible context as ours. Professors need awareness to have a general view of the class activities, to monitor the class progress, to detect problems in order to intervene in time. Students need awareness to have a view about their position in comparison to other groups. The awareness is also necessary for students to find potential collaborators to exchange documents, ideas, to ask for helps.

The eJournal enables many services that generate awareness based on fragment analysis and calculation. Besides the availability awareness such as the user presence (who is currently connecting to the environment) and the user location (local, remote-campus or remote-home condition), we also provide group awareness based on the fragment activities, which is richer than conventional artifact feedback or feed-through (Dix et al., 1993) at group and especially at community levels.

The permission-granted user can access the eJournal of others to view the annotations (if any) attached to fragments. The students can also see the group progress based on the accepted submissions. This awareness is displayed in a table, which contains the taken modules, the available modules,

\begin{tabular}{|c|c|c|c|c|c|c|c|}
\hline Group/Module & $\begin{array}{l}\text { Modélisation } \\
\text { d'un système } \\
\text { mécatronique }\end{array}$ & \begin{tabular}{|c|} 
Commande \\
numérique \\
d'un systeme \\
mécatronique
\end{tabular} & $\begin{array}{c}\text { Conception } \\
\text { dans le } \\
\text { domaine } \\
\text { frequentiel }\end{array}$ & $\begin{array}{l}\text { Module de } \\
\text { commande } \\
\text { en cascade }\end{array}$ & \begin{tabular}{|c|} 
Identification \\
par la \\
methode de \\
Levy
\end{tabular} & $\begin{array}{c}\text { Modélisation } \\
\text { d'un canal } \\
\text { aérothermique }\end{array}$ & $\begin{array}{l}\text { Commande } \\
\text { d'un canal } \\
\text { aérothermique }\end{array}$ \\
\hline $\mathrm{mt}-\mathrm{a} 12$ & $\checkmark$ & $\checkmark$ & $\checkmark$ & None & None & None & None \\
\hline $\mathrm{mt}-\mathrm{a} 13$ & $x$ & $\checkmark$ & $\checkmark$ & None & None & None & None \\
\hline mt-a01 & $\checkmark$ & $x$ & $x$ & None & None & None & None \\
\hline $\mathrm{mt}-\mathrm{a02}$ & $\checkmark$ & $\checkmark$ & $x$ & None & None & None & None \\
\hline mt-a03 & $\checkmark$ & $\checkmark$ & $x$ & None & None & None & None \\
\hline mt-a04 & $\checkmark$ & $\checkmark$ & $x$ & None & None & None & None \\
\hline mt-a05 & $\checkmark$ & $\checkmark$ & $x$ & None & None & None & None \\
\hline mt-a06 & $\checkmark$ & $\checkmark$ & $x$ & None & None & None & None \\
\hline mt-a07 & $\checkmark$ & $\checkmark$ & $x$ & None & None & None & None \\
\hline
\end{tabular}

Figure 6. The table representing the group progress based on the submission acceptance. 
and the allowed modules (Figure 6). Those are some first ways to get knowledge about their own progresses and the ones from the class.

The eJournal environment produces also charts summarizing all group's fragment (with many options such as 'since the last week', 'since the last month', or 'since the beginning of the term'). We have developed different kinds of charts such as bar chart, pie chart, or line chart. By looking at these charts, one can get an idea about the progress of each group as well as of the whole class. Our hypothesis is that the awareness could be based on the fact that 'the more students create fragments in the eJournal the more they participate in the learning processes. Figure 7 shows a bar chart, in which one can see the number of fragments ( $y$-axis) of all groups ( $x$-axis) for the whole term.

By clicking in a bar or a slice representing a group in a bar or pie chart, one can see the fragment evolution, week-by-week, during all 14 weeks of the semester (Figure 8). This information could be useful for educators to analyse some behaviors of students. For example, the peaks in the diagram representing the high number of created fragments could be explained by the fact that students work harder before the assignment due dates, especially before the laboratory test.

More discussions about the awareness support in the eJournal can be found in (Nguyen et al., 2004b).

\section{The case of mechatronics system modeling}

One of the modules that every student attending the Automatic Control course must complete is the modeling of a mechatronics system. The pedagogical objective is to visualize and characterize the dynamic behavior of an electrical drive by studying various characteristic responses in the time

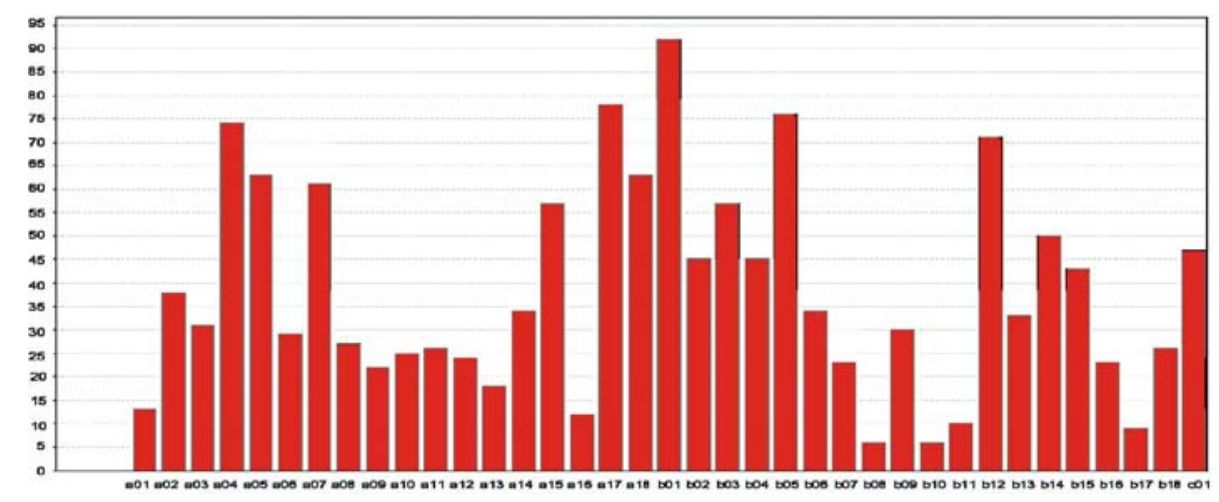

Figure 7. The bar chart representing the number of fragments of all groups for the whole term. 


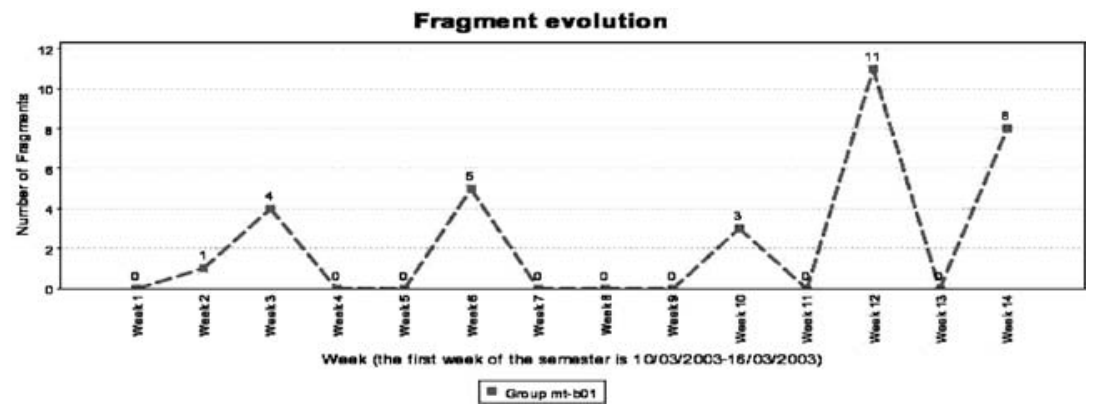

Figure 8. The fragment evolution of a group.

domain. The studies done during this session are limited to the analysis of the behavior of the drive controlled in open loop. The goal is to validate the theoretical models obtained to describe the speed evolution and position evolution. In addition to the theory reminder and the description of the experimentation environment, the cockpit composed for this experimentation assignment contains the following experimentation protocol as supplementary information.

The tasks assigned as pre-lab are the following:

1. Write a Matlab command file (M-file) that realize the identification of the static gain $(A)$ and the time constant (tau) coefficients according to the least square technique described in the theory reminder.

2. Use the provided test data to validate graphically your program, that is superpose in a same graph the test data and the step response obtained numerically using the estimated values of the static gain and the time constant.

3. Determine using an analytic method the feed forward command $(U)$ to be imposed if the desired velocity is $60[\mathrm{rad} / \mathrm{s}]$. To do this, use the typical values of the drive parameters provided in the experiment description.

The eJournal, as it has already been stated, can remotely support the whole life cycle of lab teamwork. It serves firstly as a shared workspace for the teamwork. The students start working on their pre-lab by exploring the available experimentation preparatory content included in the cockpit and the eJournal. Students study the objectives, theory and then agree how to divide the protocol tasks among them.

For example, two students of the same group, let say Alice and Bob, start their cooperation by studying the lab assignment and its objectives, and then agreeing on how to share the work between them, e.g. Alice prepares the tasks 1, and 2, and Bob prepares task 3. The two students have the flexibility 
to use the eJournal in order to work remotely on their assigned work if they wish. They document the task results, e.g. Task 1:M-file, Task 2:Values A, tau, and Graph and, Task 3: U., and then upload to the eJournal as fragments typed 'Uploaded'. The eJournal emphasizes the need of collaboration between the group members even in the preparation phase of the experimentation protocol. Students post questions to each other, e.g. Alice asks for some explanations by adding annotations to the fragments uploaded by Bob, and then Bob responds with other annotations. Bob can upload some more fragments as the reference.

After filling in the pre-lab report, Alice and Bob submit their pre-lab, which is also a fragment, from their eJournal to the Educator's eJournal. All annotations attached with the pre-lab are also sent. This could help students to explain or comment on their work.

After being granted the permissions by the TA, the students can use the remote manipulation of real setups and Web-based simulation facilities available in the cockpit to execute their lab-work tasks and then document each task's results in the eJournal. The lab-work tasks are the following:

4. Measure the actual step response of the drive velocity with a medium inertia disk and for a $15 \mathrm{~mm}$ brake position.

5. Determine with a graphical method the actual static gain $(A)$ and the time constant (tau). To achieve this goal use one of the method described in the theory reminder.

6. Estimate using the least square technique the actual static gain $(A)$ and the time constant (tau).

7. Compare the 2 methods introduced in 5 and 6 according to their ease of use and precision. Draw in the same graph the two corresponding step responses.

8. Apply to the feed forward command calculated in 3. Verify if the desired velocity is reached and analyze the possible discrepancies.

Alice and Bob can pursue different learning modalities, and work in different spaces such as Experimentation console or SysQuake Remote analysis component. The fragments in the eJournal serve as shared artifacts, which help to sustain the continuity of interaction in Space, Place, Time, and also Cognition dimensions (Nguyen et al., 2004a). The eJournal serves as a 'shared workspace' at both system (by allowing the interaction between different components within the Cockpit environment) and user levels. The lab work of Alice and Bob results in a final report being submitted to the educator.

The role of educators during the completion of modules can be very active and also important. The eJournal enables educators to observe closely how team members progress with their work by viewing the content of eJournal as well as different awareness information, and providing 
the appropriate guidance. The eJournal also enables students to request some assistance from their educators and receive responds from them. For instance, Bob sends an email with attached fragment Scale of Graph to the TA to get help.

\section{Evaluation of the eJournal}

The evaluation of the eJournal has been designed with two objectives: first to assess the collaboration activities among students and second to assess the user acceptance of the eJournal-eMersion environment. The evaluation has been based on two perspectives: a questionnaire and a $\log$ and content analysis of the eJournal (Zaphiris and Zacharias, 2001; Sire et al., 2003).

We evaluated two sets of students. The first set (Group 2002) consists of 30 students who have used the environment as part of their hands-on laboratory assignment in automatic control course during the 2002 autumn term. The students were divided into groups of two to realize 7 modules and a final practical 'lab' examination. The second set (Group 2003) consists of 96 students of Micro-engineering who have used the environment to carry out practical assignments in Automatic Control during the 2003 spring term. These students were also divided into groups of two students.

For each module the groups had to prepare first a 'pre-lab' and to submit a report to the educators and then they got access to the 'lab', i.e. they got either local or remote access to the physical devices for experimentation. For both the 'pre-lab' and the 'lab' part of their work, students could choose between face-to-face and flexible learning modalities. The teaching assistants were available during planned face-to-face sessions in the laboratory room. The students were free to progress at their own pace, however an indicative schedule was suggested to the groups.

\subsection{QUESTIONNAIRE ANALYSIS}

A user-interface satisfaction questionnaire - the Computer System Usability Questionnaire (CSUQ) (Lewis, 1995; Perlman, 2002) - was the main instrument used to assess the acceptance of the eJournal-eMersion environment by its users. This is a Likert scale questionnaire made of nineteen usability related assertions to which the respondent has to agree or disagree using a seven-point scale, ranging from 1 (strongly disagree) to 7 (strongly agree). The questionnaire also comprises of open fields for listing the three most positive and negative aspects of the tool and open fields for comments. These six open questions were very useful in revealing important information and details about collaboration and general flexible uses of the environment by the students. 
The CSUQ questionnaire has been extended with 7 factual questions (name, surname, sex, electronic courses taken, familiarity with computers, questions about students' own computer if $\mathrm{s} /$ he has any, perceived eJournal use). Some of these questions such as familiarity with computers (ranging from 1, very low, to 7, very high) and the perceived eJournal use (ranging from 1 to 5 as compared to the 4 other components of the eMersion environment) have been included for checking if they have an influence on students' satisfaction. The other factual questions such as student's identity have been included to personalize log analysis.

Overall, the sample of the questionnaire evaluation consists of 93 students, i.e. 22 students (out of 30) from Group 2002 and 71 (out of 96) from Group 2003. Concerning the gender of the sample, all the 2002 students were males, and for the 2003 group 67 were male $(94.4 \%)$ and 4 female $(5.6 \%)$. Due to very small presentation of female in the sample no gender differentiations would be attempted. Other background information of the subjects revealed that an overall percentage of $82 \%$ of the students reported they have a computer connected with the Internet. This percentage is quite higher for the 2003 (59 students, 83\%) compared to the 2002 group (77\%). Concerning the reported 'familiarity with computers' the majority of the students in both groups (77\% of the 2002 Group and $76 \%$ of the 2003 Group) rated their familiarity as high, using 5, 6, and 7 of the rating scale provided.

\subsubsection{Analysis of the statements}

The reliability of the 19 statements of the questionnaire is high, with reliability coefficient (i.e. Cronbach alpha) 0.88. Principal Components Factor analysis on the 19 statements of the CSUQ questionnaire revealed that the statements can be clustered into 5 constructs/factors which appear to be theoretically meaningful:

- Factor 1: "Easiness of the system" (4 statements)

- Factor 2: "Completion of my work" (4 statements)

- Factor 3: "Information retrieval" (5 statements)

- Factor 4: "System Interface" (4 statements)

- Factor 5: "Error Messages/Mistake Recovery" (2 statements)

The total variance explained by these five factors is quite high (i.e. $70.3 \%$ ). A comprehensive list of the above five factors together with the statements they consist of, and the factor loadings of each statement, is shown in Appendix 1. The last column of the tables in the Appendix also presents the overall percentage of agreement to each evaluation statement of the whole sample $(N=93)$.

The following graph (Figure 9) presents the total percentages of the students who rated positively these factors. 


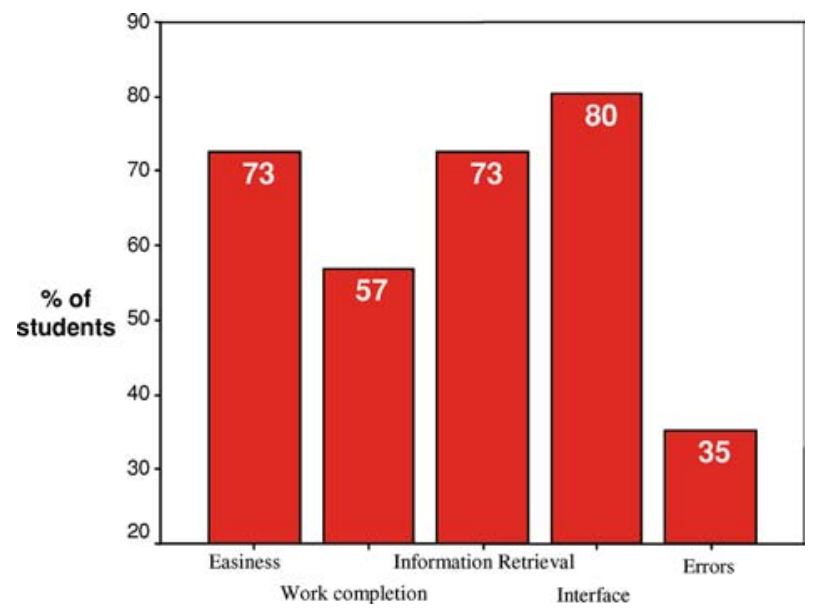

Figure 9. Percentages of the Students $(n=93)$ who rated positively each factor.

As it can be seen from the above graph, the 'system interface' was the most positively rated factor since $80 \%$ of the students gave a high rating (agreement) to the statements that dealt with 'system interface' in the questionnaire. The easiness of the system and statements related to 'information retrieval' using the tool appear to have the second place in positive ratings (both $73 \%$ of agreement) with the impact of the tool in aspects related to work completion coming next ( $57 \%$ of agreement). The lowest scores were given to the two statements that constitute the 'Error Messages and Mistake Recovery' factor. No significant differences were found in the responses of the two classes concerning their ratings of the evaluation factors.

Analysis also revealed that the statements of the factor 'easiness of the system' (Q1, Q2, Q6 and Q7) as well as statement 14 are correlated with the variable 'familiarity with computers', with the correlation coefficients presented in the following Table.

Since all correlations are positive, this implies that the more familiar the users reported to be with computers the more they tended to agree with statements that evaluate the perceived "easiness" of the system (i.e. they find it easier), or they found the information effective in helping them complete their tasks (Q14). It also implies that students who are less familiar with computers encounter some difficulties in using the environment. That means that in future redesign of the tool we need to consider these issues and improve even further the system's help facilities, error messages and user interface in general.

\subsubsection{Analysis of the open questions}

Additional information from the questionnaire involves the open questions at the end, where the students were asked to report the three most positive (in 
Table I. Statements with statistically significant correlation with the variable familiarity with computers'

\begin{tabular}{lll}
\hline Statement & $\begin{array}{l}\text { Correlation } \\
\text { coefficients } \\
\text { (Non parametric) }\end{array}$ & Significance \\
\hline Q1: Overall, I am satisfied with how easy it is to & 0.320 & $* *$ \\
$\quad$ use this system & 0.370 & $* *$ \\
Q2: It was simple to use this system & 0.378 & $* *$ \\
Q6: I feel comfortable using this system & 0.227 & $*$ \\
Q7: It was easy to learn to use this system & 0.269 & $* p<0.05$ \\
Q14: The information is effective in helping me & & $* * p<0.01$ \\
& & \\
\end{tabular}

order of importance) and the three most negative aspects of the eJournal. This section presents the results of this analysis. The responses of the students were firstly listed and then organized into categories based on their context. Concerning the three most positive aspects, students commented mainly on the categories presented in the following Table.

As it can be seen on the following Table, the most frequently positively commented aspect of the system was its flexibility. Students enjoy and find useful the fact that the system can be used 'anytime' and from 'anyplace'. The integration of all the necessary tools (e.g. cockpit for remote experimentation, eJournal for remote collaboration, Remote SysQuake for remote data analysis, etc.) for the completion of their work in one environment (i.e. eMersion) appears second in students' positive comments (quoted from

Table II. The positive comment categories (their frequencies and percentages)

\begin{tabular}{|c|c|c|c|c|c|c|c|}
\hline \multirow[t]{2}{*}{ Category } & \multicolumn{2}{|l|}{ Positive 1} & \multicolumn{2}{|l|}{ Positive 2} & \multicolumn{2}{|l|}{ Positive 3} & \multirow[t]{2}{*}{ Total $(\%)$} \\
\hline & Frequency & $\%$ & Frequency & $\%$ & Frequency & $\%$ & \\
\hline Flexibility & 29 & 48.3 & 5 & 17.2 & 1 & 25 & $35(37.6)$ \\
\hline $\begin{array}{r}\text { Integration of all } \\
\text { necessary tools }\end{array}$ & 7 & 11.7 & 9 & 31 & 1 & 25 & $17(18.4)$ \\
\hline Collaboration & 4 & 6.7 & 7 & 24.1 & & & $11(11.8)$ \\
\hline Easy to use & 7 & 11.7 & 2 & 6.9 & & & $9(9.7)$ \\
\hline Interface & 5 & 8.3 & 3 & 10.3 & & & $8(8.6)$ \\
\hline Cool/Interesting & 7 & 11.7 & 1 & 3.4 & & & $8(8.6)$ \\
\hline Info/Theory & 0 & & 1 & 3.4 & 2 & 50 & $3(3.2)$ \\
\hline SysQuake & 1 & 1.7 & 1 & 3.4 & & & $2(2.1)$ \\
\hline Total & 60 & 100 & 29 & 100 & 4 & 100 & $93(100)$ \\
\hline
\end{tabular}


students' responses, the phrase most frequently used is 'all in one'). Students also enjoyed the different collaborative features provided by the eJournal, such as exchange of messages, knowledge, information and objects and group awareness. Students also reported positive comments concerning the system's interface, easiness of use and other aspects that appear in the Table with lower frequencies.

The negative aspects of the system as perceived by the students were also analyzed. The following Table shows the categories as emerged from the comments of the students.

The majority of the total 76 'negative' comments (around 80\%) can mainly be clustered into the first two categories presented in the following Table: (a) Technical limitations (the system is down sometimes, slow from home, and still has some bugs) and (b) eJournal interface (the system does not provide satisfactory help, feedback, FAQ, and error messages). Other comments, less frequent, revealed other drawbacks as perceived by the students, like the limitation of real time interaction and the absence of contact with educators when working remotely. Those comments of the students are of great importance and will be taken into consideration for the improvement of the system.

\subsection{LOG AND CONTENT ANALYSIS}

The second aspect of the eJournal evaluation relies on the analysis of the content of the database that holds the shared workspace. This database contains the fragment meta-information and some logs of student's activities such as when and which fragments they viewed, copied, moved, etc.

\subsubsection{Quantitative analysis of $\log$ data}

The first set of students created a total of 1412 fragments. This gives a mean of 88 fragments per group, or if we consider the 7 modules and the final practical, of about 11 fragments for each module. As one of these fragments is supposed to contain the "pre-lab" report, we can consider that each group

Table III. The negative comment categories (their frequencies and percentages)

\begin{tabular}{|c|c|c|c|c|c|c|c|}
\hline \multirow[t]{2}{*}{ Category } & \multicolumn{2}{|l|}{ Negative 1} & \multicolumn{2}{|l|}{ Negative 2} & \multicolumn{2}{|l|}{ Negative 3} & \multirow[t]{2}{*}{ Total $(\%)$} \\
\hline & Frequency & $\%$ & Frequency & $\%$ & Frequency & $\%$ & \\
\hline Technical Limitations & 20 & 42.6 & 7 & 35 & 3 & 33.3 & $30(39.5)$ \\
\hline Interface/Complex & 17 & 36.2 & 8 & 40 & 6 & 66.7 & $31(40.8)$ \\
\hline Contact with Assistants & 5 & 10.6 & 2 & 10 & & & $7(9.1)$ \\
\hline Real Time Interaction & 3 & 6.4 & 1 & 5 & & & $4(5.3)$ \\
\hline SysQuake Remote & 2 & 4.2 & 2 & 10 & & & $4(5.3)$ \\
\hline Total & 47 & 100 & 20 & 100 & 9 & 100 & 76 \\
\hline
\end{tabular}


created about 10 fragments in performing each module. These fragments are messages, sharing and storage of information. The analysis of these logs also shows that $75.99 \%$ of all the fragments were produced within the environment. The fragments added in the eJournal outside of face-to-face sessions represent $26.63 \%$ of all the fragments. It's significant to note that every week some students added fragments 1 or 2 days before the face-to-face sessions.

We have examined the type of fragments created during flexible work (i.e. outside of face-to-face sessions) to determine the type of work students were performing without the presence of assistants. The graph of Figure 10 shows that $47 \%$ of these fragments are text files that most likely correspond to the writing of a report for the 'pre-lab' activities. The $37 \%$ of image files and the $4 \%$ of mathematical scripts suggest that students did use other software tools and imported their results, to share images for writing a report for instance, or to execute the scripts in the mathematical console provided with the environment.

The analysis of the second group logs shows that every group created a mean of 36 fragments in performing the practical modules for the whole semester, or if we consider the 3 modules, of about 12 fragments for each module. This means that all groups created a significant amount of fragments. Eighty-six percent of the fragments were created within the environment with the Experimentation component and the SysQuake Remote; the remaining $14 \%$ were fragments created with external applications. The number of fragments created in flexible sessions (occurs outside the laboratory) was $55 \%$. This means that students accepted and already worked in different learning modalities. Figure 10 shows the types of fragments created during students' flexible work.

The mean number of fragments that each student made to each module was also correlated with the statements of the satisfaction questionnaire. This analysis revealed a statistically significant correlation between the number of
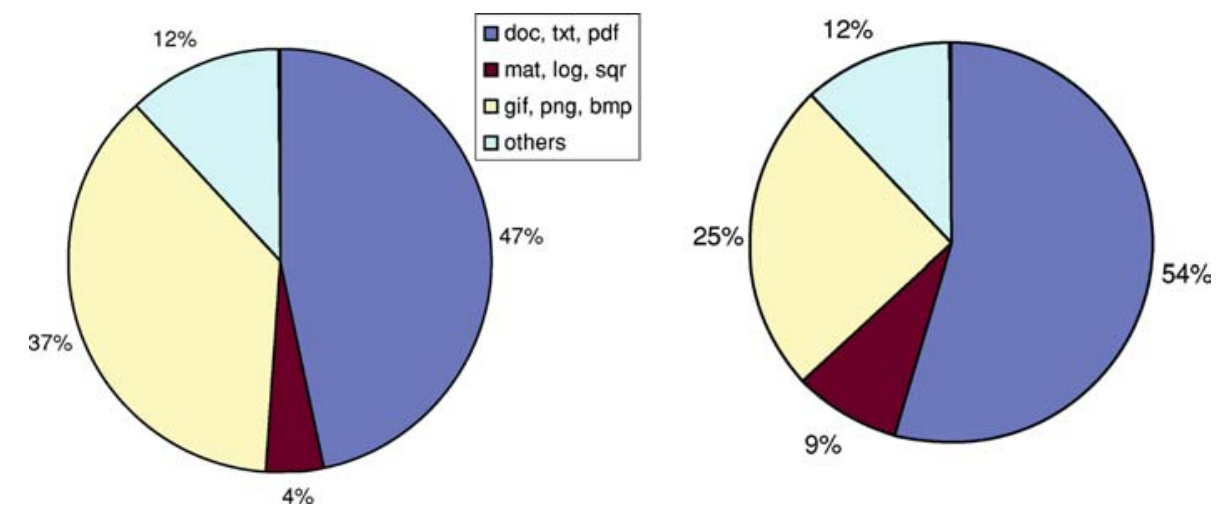

Figure 10. Types of flexible fragments (Group 2002; Group 2003). 
fragments and Q7 "It was easy to learn to use this system" (Pearson correlation coefficient $=0.293, p<0.05$ ). The positive value of the coefficient implies that the students who had more involvement with the system (more fragments) tend to find it easier to learn using it.

\section{Discussion}

We found the results above satisfactory concerning the acceptance of the tool as the questionnaire shows that most of the students say they were satisfied. However, as our questionnaire was not anonymous, we can wonder if it had an influence on student's answers. In most of the emerged factors students responded positively (with percentages over 55\%). Students show dissatisfaction regarding only two statements i.e. technical limitations and user interface. These findings are very useful for future improvements of the system.

Students comments on the three open questions of the questionnaire support our expectations that the tool supports them to work in a flexible manner any time from anywhere (even from their home during the weekend). The 'flexibility' aspect of the tool is the students first favor feature of the eJournal environment. The $\log$ data shows that $26.63 \%$ and $55 \%$ of the fragments created by Group 2002 and Group 2003 respectively were flexible fragments. That shows that flexible learning modalities have been chosen, and this independently from the fact that we estimated that the students had enough time to perform all the work in face-to-face modalities. The significant increase of flexible fragments by Group 2003 is because this group, from Micro-engineering section, preferred for practical reasons to use their own computer labs equipped with PCs.

The questionnaires also revealed that students were satisfied with the collaborative and cooperative facilities of the eJournal environment. This is also confirmed by the eJournal log data. We found students participation and collaboration encouraging as all the groups created a significant amount of fragments. Each group created in average 11-12 fragments for each experimentation module. These fragment's content ranges from discussion fragments, exchange of knowledge, recording and sharing of outcomes, assistance from educators, to submission of work.

\subsection{CONCLUDING REMARKS AND FURTHER WORK}

In this paper, an Electronic Laboratory Journal (eJournal) is proposed as a collaborative and cooperative paradigm for remote experimentation in engineering education. The eJournal facilitates a group of students to work 
remotely in order to complete their experimentation assignments by discussing, exchanging or sharing information and also by documenting and finally submitting to their educators their results.

In this paper, the application and evaluation of the eJournal paradigm in the framework of the eMersion project are presented. The evaluation results of the eJournal were very satisfactory. The evaluation was based on questionnaires and eJournal log data analysis. The evaluation shows that students accepted the environment and they enjoyed the flexible and collaborative features of the environment.

Further work includes the improvement of the system based on students' feedback, i.e. improvement of the interface (error messages, mistake recovery, help, FAQ) and the technical limitations (system availability and performance). Further research along this line would address a more intelligent interchange of information between the eJournal and the eMersion cockpit (or any other remote experimentation environment), i.e. production of experimentation results by the cockpit in a structured format that will be understandable by the eJournal (the XML infrastructure is ideal for this purpose).

\section{Acknowledgements}

This work is funded by the Board of the Swiss Federal Institutes of Technology in the framework of its New Learning Technologies (NLT) program and by the Swiss National Science Foundation under grants number 510.407. The authors would like to cordially thank Maria Pampaka for her support and contribution in this work.

\section{Appendix I}

\begin{tabular}{lll}
\hline Statements in each factor & $\begin{array}{l}\text { Factor } \\
\text { Loadings }\end{array}$ & $\begin{array}{l}\% \text { of } \\
\text { students } \\
\text { who agree }\end{array}$ \\
\hline Factor 1: "Easiness of the system" & & \\
Q1: Overall, I am satisfied with how easy it is to use this system & 0.55 & $73 \%$ \\
Q2: It was simple to use this system & 0.77 & $76 \%$ \\
Q6: I feel comfortable using this system & 0.74 & $61 \%$ \\
Q7: It was easy to learn to use this system & 0.76 & $76 \%$ \\
Factor 2: "Completion of my work" & & \\
Q3: I can effectively complete my work using this system & 0.70 & 75 \\
Q4: I am able to complete my work quickly using this system & 0.76 & 51 \\
Q5: I am able to efficiently complete my work using this system & 0.82 & 55 \\
Q19: Overall, I am satisfied with this system & 0.60 & 73 \\
\hline
\end{tabular}


Appendix I Continued.

\begin{tabular}{lll}
\hline Statements in each factor & $\begin{array}{l}\text { Factor } \\
\text { Loadings }\end{array}$ & $\begin{array}{l}\% \text { of } \\
\text { students } \\
\text { who agree }\end{array}$ \\
& & \\
\hline $\begin{array}{l}\text { Factor 3: "Information retrieval" } \\
\text { Q8: I believe I became productive quickly using this system }\end{array}$ & 0.52 & 57 \\
Q11: The information provided with this system (such as \\
$\quad \begin{array}{l}\text { online help, on-screen } \\
\text { messages, and other documentation) is clear }\end{array}$
\end{tabular}

\section{References}

Agostinho, S., G. Lefoe and J. Hedberg (2001): On-line Collaboration for Effective Learning: A Case Study of a Postgraduate University Course, AusWeb01, the Seventh Australian WWW Conference, April 21-25.

Appelt, W. and P. Mambrey (1999): Experiences with the BSCW Shared Workspace System as the Backbone of a Virtual Learning Environment for Students, In Proceedings of ED-MEDIA 1999, Seattle, USA, pp. 1710-1715.

Armstrong, B. and R. Perez (2001): Controls Laboratory Program with an Accent on Discovery Learning. IEEE Control Systems Magazine vol. 21, no. 1, pp. 14-20.

Arzberger, P. and T.A. Finholt (2002): Data Collaboratories in the Biomedical Community, A Report of a panel meeting held in September, available at nbcr.sdsc.edu/Collaboratories/ CollaboratoryFinal2.doc

Atkins, D.E., K.K. Droegemeier, S.I. Feldman, H. Garcia-Molina, M.L. Klein, D.G. Messerschnmitt, P. Messina, JP. Ostriker and M.H. Wright (2003): Revolutionizing Science and Engineering through Cyberinfrastructure, Report of the National Science Foundation blue-ribbon advisory panel of cyberinfrastructure, available at http://www.communitytechnology.org/nsf_ci_report/ 
Berge, Z.L. and M. Collins (1995): Computer-Mediated Communications and the Online Classroom: An Introduction. In Z.L. Berge and M.P. Collins (eds.): Computer Mediated Communication and the Online Classroom Vol. 1, Overview and Perspectives. New Jersey: Hampton Press Inc., pp. 1-10.

Berman, H.M., Z. Westbrook, Z. Feng, G. Gilliland, T.N. Bhat, H. Weissing, I.N. Shindyalov and P.E. Bourne (2000): The Protein Data Bank. Nucleic Acids Research vol. 28, 235-242.

Böhne, A., N. Faltin and B. Wagner (2004): Synchronous Tele-tutorial Support in a Remote Laboratory for Process Control. In Innovations 2004: World Innovations in Engineering Education and Research (pp. 317-329), iNEER in cooperation with Begell House Publishers.

Dix, A., J. Finlay, G. Abowd and R. Beale (1993): Human Computer Interaction. Prentice Hall. 1993.

Dourish, P. and V. Bellotti (1992): Awareness and Coordination in shared workspaces. Proceedings of CSCW'92, Toronto, Canada, 107-114.

Eastmond, D. and J. Ziegahn (1995): Instructional Design for the Online Classroom. In Z.L. Berge and M.P. Collins (eds.): Computer Mediated Communication and the Online Classroom Volume Three: Distance Learning. E, New Jersey: Hampton Press Inc., pp. 29-36.

Geist, A. and N. Nachtigal (2001): ORNL Electronic Notebook Project, 2001, available over the Internet http://www.epm.ornl.gov/ geist/java/applets/enote/

Gillet, D., C. Salzmann, H.A. Latchman and O.D. Crisalle (2000): Advances in Remote Experimentation, American Control Conference, Chicago, USA, June 28-30, pp. 2955-2956.

Gillet, D. and G. Fakas (2001): eMersion: A New Paradigm For Web-Based Traning In Engineering Education, International Conference on Engineering Education (ICEE), Oslo, Norway, pp. 10-14.

Gillet, D., F. Geoffroy, K. Zeramdini, A.V. Nguyen, Y. Rekik and Y. Piguet (2003): Cockpit: An effective metaphor for Web-based Experimentation in Engineering Education. International Journal of Engineering Education vol. 19, no. 3, pp. 389-397.

Gillet, D. (2003): Towards Flexible Learning in Engineering Education. In Engineering Education and Research-2003: A Chronicle of Worldwide Innovations, published by iNEER in cooperation with Begell House Publisher, pp. 95-102.

Gomez, L.M. and D.N. Gordin (1996): Establishing Project Enhanced Classrooms through Design. In D. Jonassen and G. McCalla (eds.): Proceedings of ICCE '95 International Conference on Computers in Education, Charlottesville, VA: Association for the Advancement of Computing in Education, pp. 20-27.

Gutwin, C., M. Roseman and S. Greenberg (1996): A Usability Study of Awareness Widgets in a Shared Workspace Groupware System. Proceedings of CSCW'96, Massachusetts, pp. 258-267.

Foster, I. (2002): The Grid: A New Infrastructure for 21st Century Science. Physics Today vol. $55,42-50$.

Harasim L. (1989): On-Line Education. A New Domain. In R. Mason and A. Kaye (eds.): Mindweave: Communication, Computers and Distance Education, Oxford: Pergamon Press, pp. 50-62.

Jonassen, D., M. Davidson, C. Collins, J. Campbell and B.B. Haag (1995): Constructivism and Computer-Mediated Communication in Distance Education. The American Journal of Distance Education vol. 9, no. 2, pp. 7-26.

Klöckner, K. (2000): BSCW-Educational Servers and Services on the WWW: How shared Workspaces Support Collaboration in Educational Projects. Australia: International Distance Education and Open Learning Conference. 
Kouzes, S.T., J.D. Myers and W.A. Wulf (1996): Collaboratories: Doing science on the Internet. IEEE Computer vol. 29, no. 8, pp. 40-46.

Latchman, H.A., Ch. Salzmann, S. Thottapilly and H. Bouzekri (1998): Hybrid Asynchronous and Synchronous Learning Networks in Distance Education. Rio de Janeiro Brazil: International Conference on Engineering Education.

Leifer, L. (1997): Design Team Performance: Metrics and Impact of Technology. Evaluating Corporate Training: Models and Issues: Kluwer.

Lewis, J.R. (1995): IBM Computer Usability Satisfaction Questionnaires: Psychometric Evaluation and Instructions for Use. International Journal of Human-Computer Interaction vol. 7 , no. 1 , pp. $57-78$.

Martínez, A. et al. (2002): Studying Social Aspects of Computer-supported Collaboration with a Mixed Evaluation Approach. Proceedings of the CSCL'2002, Boulder, USA.

McCormack, J.B., R.K. Morrow, H.F. Bar, R.J. Burns and J.L. Rasmussen (1991): The Complementary Roles of the Laboratory Notebooks and Laboratory Reports. IEEE Transactions on Education, vol. 34, no. 1.

ME310 (2003): The me310 Team Based Design Innovation With Corporate Partners, me310.Stanford.edu.

Myers, J.D, C. Fox-Dobbs, J. Laird, D. Le, D. Reich and T. Curtz, (1996): Electronic Laboratory Notebooks for Collaborative Research. In Proceedings of the 5th Workshop on Enabling Technologies: Infrastructure for Collaborative Enterprises, pp. 19-21.

Myers, J., A.R. Chappel, M. Elder, A. Geist and J. Schwidder (2003): Re-Integrating the Research Record. Computing in Science and Engineering vol. 5, no. 3, pp. 44-50.

Myers J. (2003): Collaborative Electronic Notebooks as Electronic Records: Design Issues for the Secure Electronic Laboratory Notebook (ELN), Proceedings of the Fourth International Symposium on Collaborative Technologies and Systems, Orlando, Florida, vol. 35, no. 1, pp. 13-22.

Nguyen, A.V., D. Gillet, Y. Rekik and S. Sire (2004a): Sustaining the Continuity of Interaction in Web-based Experimentation for Engineering Education. In Proceedings of the International Conference on Computer Aided Learning In Engineering Education, Grenoble, France, February 16-18, 2004.

Nguyen, A.V., D. Gillet and S. Sire (2004b): Sustaining Collaboration within a Learning Community in Flexible Engineering Education. In Proceedings of the 16th AACE World Conference on Educational Multimedia, Hypermedia \& Telecommunications (ED-MEDIA), Lugano, Switzerland, June 21-26.

Olson, G., D. Atkins, R. Clauer, T.A. Finholt, F. Jahanian, T. Killeen, A. Prakash and T. Weymouth (1998): The Upper Atmospheric Research Collaboratory (UARC). $A C M$ Interactions vol. 5, no. 4, pp. 48-55.

Olson, G., S. Teasley, M. Bietz and D. Cogburn (2002): Collaboratories to Support Distributed Science: The Example of International HIV/AIDS Research, Proceedings of SAICSIT 2002, pp 44-51.

Perlman G. (2002): Web-Based User Interface Evaluation with Questionnaires, http:// www.acm.org/ perlman/question.html

Roschelle, J. et al. (1999): Developing Educational Software Components, IEEE Computer Magazine, September 1999, pp. 50-58.

Schmid, C. (1999): A Remote Laboratory Using Virtual Reality on the Web, Simulation, Vol. 73, No. 1, July 1999, pp. 13-21.

Sire, S., A.V. Nguyen and D. Gillet (2003): Evaluation of a Web-based Training Environment for Hands-on Experimentation, International Conference on Engineering Education, Valencia, Spain, July 2003. 
Tan, K.K., T.H. Lee and F.M. Leu (2000): Development of a Distant Laboratory using LabVIEW. The International Journal of Engineering Education vol. 16, no. 3, pp. 273-282. The Learning through Collaborative Visualization (CoVis) project, Project's Home page available over the Internet: http://www.covis.nwu.edu/info/

Zaphiris, P. and G. Zacharias (2001): Usability Evaluation of an On-Line Modern Greek Language Course, In Proceedings of the 8th Panhellenic Conference on Informatics, Nicosia, Cyprus, November 8-10. 